\title{
Plain language for interpreting in consulting rooms
}

\author{
HM Lesch, DLitt \\ Department of Afrikaans and Dutch, University of Stellenbosch
}

Key words :

Interpreting, intercultural communication, plain language, health care, power gap

Correspondence address:

Harold M Lesch

University of Stellenbosch

Private Bag X1,

Matieland, 7602

Tel : (021) 808-3573

Fax : (021) 808-3581

E-mail : Hlesch@sun.ac.za

\section{Abstract: Curationis 30(4): 73-78}

Intercultural communication is by nature a complex activity. In a multilingual society like ours, it will inevitably surface in the health care sector. The services of an interpreter are often considered to break the impasse in this communication process. The communication problem between the two parties, the service provider and client/ patient, is often not simply a matter of language but societal factors of which the liaison interpreter should be aware of also plays a major role for effective extended communication.

This article focuses on some of the problems in rendering an oral source text in multilingual and multicultural societies such as South Africa in which there are heterogeneous target audiences for interpreting. It is pointed out that interpreters in such societies must take into account the heterogeneity of the target audiences, or otherwise interpreting will only be symbolic gestures, empty of value, and thus not communicate the message intended. In the process the limitations of the interpreter and how the presence of the interpreter can be facilitated, is also highlighted.

\section{Introduction}

Intercultural communication is a complex undertaking - more so in the South African context as a result of the previous political policies, which led to cultural and linguistic isolation between various communities. The problem of communication between two parties is often not simply a matter of language; it is equally created and compounded by the fact that the two parties are separated by a wide gap of power. This power gap is directly related to class, race and/or culture, often to gender, and to the differential power relations between a professional and his or her lay client. The need for an interpreter is realised in such an intercultural context, and the role of an interpreter as a cultural broker becomes invaluable.

For the linguistically ill-informed, the linguistic problem that arises is often solved by the mere provision of an interpreter. This is a step in the right direction, but should be enhanced by alerting the different parties to the complex nature of the linguistic activity that is about to take place and to the limitations of the interpreter as a human being.

The article is sub divided as follows: firstly the objectives and the rationale for the review is stated; secondly the intercultural reality with reference to the difficulties occurring when interpreting in health care is highlighted; thereafter the mental efforts are considered from the viewpoint of the interpreter and how it can be eased during the interpreting process; and finally one concludes by complementing the availability of the interpreter.

The aims of this article are -

i) to consider from the viewpoint of the interpreter the complex nature of intercultural communication during consultation with reference to 
the Western Cape; and to discuss the limitations of the interpreter with reference to the mental efforts of interpreting and to determine in which manner the presence of the interpreter can be facilitated.

\section{The rationale}

This review sets out to characterize the uniqueness of interpreting in the health care to gain a better understanding and insight into the phenomenon. Furthermore, the motivation for the review also flows from the fact that most of the research on language barriers originates in developed countries that experience an influx of people from less affluent countries, and are encountering new difficulties with communication related to language and culture (Schlemmer, 2005: 2-3). In South Africa the major problem is not encountered with immigrants but with fellow South Africans. The latter is a concern more so in South Africa because it seems rare to have personnel dedicated to interpreting.

As part of the implementation strategy of the Western Cape language policy that was launched in February 2005, it is proposed that professional health care interpreter posts need to be progressively established in each hospital to provide quality health care interpreting services (Cole, Lawrence, Nyubuse \& Godden 2003:42-43). The reality is that there are very few professional interpreters and isiXhosa speaking nurses, and general assistants are often called upon to assist; sometimes even the patients' relatives are requested to interpret. This is confirmed by the Emzantsi report (Cole, et al 2003:28-29), which states that the biggest demand for interpreting services is clearly in the Department of Health, where the need for health workers and patients to understand one another is often a matter of life and death.

\section{The South African reality}

Interpreting becomes particularly informative and worthwhile when distinctive languages and cultures are involved. Speech (as well as writing) is a culturally constructed act that includes various aspects, such as socialisation and gender, while the linguistic system is part of all the other systems of culture. One should also think of interpreting as the reproduction of culture, since it transfers certain aspects of culture belonging to one group to those of another. Along these lines, one should think of language in culture and not just language and culture (Fourie 2003:36). Many cultural groups, particular those coming from or living in rural areas, will for instance, have difficulty in understanding an interview conducted via a learned corpus that is not suited to their educational level or background.

Since the realisation of the new democratic dispensation in South Africa in 1994, cross-cultural cooperation has been responsible for the development of the intercultural 'rainbow nation'. Repeated cross-cultural communication among the same people could eventually create an interculture with its own norms (Fourie 2003:38).

As a starting point, one can take the statistics (addendum A) regarding the level of education and that of the cultural groupings of the Western Cape (addendum B). Regarding language and health care, it involves the fundamental principle that the ordinary citizen should not only have access to health care facilities. The opposite side of this principle is often that the ordinary citizen should converse and make his health problem known. A proper understanding of the illness arises through communication, whether it is by the health care practitioner or institutions entrusted with the responsibility for health care. This communication is by no other means than language.

According to the latest census figures, South Africa currently has an estimated population of 45 million. Yekiso (2004:7) points out that $70 \%$ of this population speak indigenous African languages. It is a population characterised by multilingualism and multiculturalism. Yet, despite this linguistic and cultural diversity, English and Afrikaans are still the sole languages used in trials and the keeping of court records. A significant segment of the population still finds itself in the tentacles of a language barrier in so far as court proceedings are concerned. A study carried out by Viljoen and Nienaber (2001:121-135) shows that there is overwhelming support for the use of accessible language in a legal context from both legal professionals and clients. Unfortunately, the practice often shows the opposite. It also shows that it takes less time to understand a message that is conveyed via accessible language and that there are a greater percentage of correct responses.

The reality of the situation in South Africa is that there are eleven official languages, nine of which are indigenous languages and the other two being English and Afrikaans. The nine indigenous African languages, in turn, have several dialects that are not necessarily mutually intelligible. The decision to have eleven official languages may have been prompted more by a need to heal the divisions of the past and the need to build a united and democratic South Africa than on the basis of considerations of practicality.

The mentioned statistics are also relevant to intercultural communication as it relates to liaison (including community) interpreting. The role of the liaison interpreter is to facilitate communication between a public service provider (i.e. doctor, (para) legal professional) and a user of that service who does not share the same language or culture. In this case he or she is called a liaison interpreter because the starting point is recognition that the problem of communication between these two parties is not simply a matter of language or culture. It is compounded by the fact that they are separated by a wide gap of power. This power discrepancy between a professional and his or her lay client could contribute to ineffective communication. The liaison interpreter should be more proactive in the sense that he or she should not only interpret for the clients, but should represent their interests, assess their needs and help them obtain whatever they are entitled to.

In urban South Africa today, the clients/ patients requiring these services are usually those who are most disadvantaged in terms of education, location and socio-political power. They are often the socially marginal or precarious inhabitants of the most economically deprived, far-flung, urban squatter areas, and include the rural newcomer, the very old and young, and those with fewer employment possibilities (Muller 1996:56; Crawford 1994; Swartz 1992:11-13 and Lesch 1999:113-130).

The situation is further complicated for the interpreter by the mere fact that languages have several registers, e.g. 
specialist, conversational, etc., which are generally defined as the social/intellectual level of the speaker. When interpreting in a medical context, the interpreter must be able to determine the patient's register and communicate with him or her on that level. Otherwise, the interpreter runs the risk of alienating the patient or promoting the 'nodding syndrome', that is the patient will nod in agreement out of fear or embarrassment without understanding what is being said. Health professionals and interpreters need to be aware of the quick nod, because many of the questions posed in their context require 'yes' or 'no' answers.

Some patients may have a complete absence of register with regard to certain subjects. Patients may have great difficulty in discussing gynaecological problems, for instance; many women have never given names to body parts such as the vagina, or to aspects of sexual activity such as 'single partner', 'multiple partners', or even 'sexual intercourse'. The interpreter must therefore be clear and diplomatic at the same time. Despite her best efforts, the patient may still provide the wrong information, none at all, or simply succumb to embarrassment (Ergueta 1992:12).

In order to overcome such culturallybased difficulties, it is helpful for the interpreter to meet the patient before the interview, so the interpreter can determine the patient's educational background, attitudes toward health care and other aspects of his or her social background. The interpreter will then know which register is most appropriate for that particular situation. Even a bicultural interpreter may have difficulty in identifying and translating many of the terms used because of an abundance of dialects in a given language. The patient may not be able to explain what actually took place during an accident or which symptoms appeared first unless the interpreter encourages the patient to speak more freely. This may take time.

English-speaking health care professionals tend to speak a jargon of their own. There are two possibilities for overcoming this: either the interpreter should take responsibility for simplifying the medical language, or the doctors and nurses should make an effort to use everyday expressions. Experience has shown that the second approach is preferable. Otherwise, the interpreter may have to interrupt frequently to ask for an illustration of a point before it can be interpreted.

Often, patients who have learned the meaning of a word in one context tend to apply it in other contexts, often wrong ones. Thus, one patient who was told that she was to be "discharged tomorrow" concluded that she was going to develop "a discharge from below". Doctors and nurses may want to have an idea of the patient's perception of the problem, state of mind, educational background, selfimage or attitude towards health. Nuances may be lost, and statements misinterpreted, if the interpreter tries to polish the patient's language. This is also why summarising is dangerous, especially in a psychiatric setting (Ergueta 1992:12).

Many patients tend to add irrelevant material because it lessens their feeling of embarrassment. Often, in answering health-related questions, they are more comfortable if the attention is not focused on their medical problem, especially if it is of a 'personal' nature. Cultural factors may also come into play: many patients believe that health is directly related to things such as the weather, the environment and certain eating habits. Thus, what may appear to be irrelevant information is, from their point of view, highly relevant. If they are prevented from giving a full account of such circumstances, they may conclude that no one is interested in their case. They may even become 'alienated' and lose confidence in the medical environment. For all these reasons, it is usually better for the interpreter not to cut the patient short and to render faithfully all seemingly irrelevant information.

The non-verbal aspects of communication, e.g. intonation patterns, facial expressions and gestures, tell their own story. It is important for all involved in the interview to be able to see, as well as to hear, each other. Interpreters who 'act out' their message are likely to be more effective in communicating. They should be aware, however, of the differences in body language of different cultures.

Research carried out by the now defunct National Language Project in the early 1990s (Ntshona 1999:144-150) showed clearly that there is a crisis in the health service in and around Cape Town, where the English- or Afrikaans-speaking health care providers and the isiXhosa-speaking patients do not understand one another. There is reason to believe that the situation has not changed dramatically if one looks at the statistics - limited inroads have been made. The reality is that there are no professional interpreters and isiXhosa-speaking nurses, and general assistants are often called upon to assist; sometimes even the patients' relatives are requested to interpret. This is confirmed by the Emzantsi report (Cole et al 2003:28-29), which states that the biggest demand for interpreting services is clearly in the Department of Health, where the need for health workers and patients to understand one another is often a matter of life and death.

The aforementioned report confirms the following (Cole et al. 2003:29):

- There are no official interpreters in health (Western Cape), and the main need is for interpreting between isiXhosa speakers and non isiXhosa health workers.

- The main source of interpreters at present is nurses, nursing assistants, auxiliary staff and community volunteers. The interpreting is entirely informal. It is mainly the nursing staff who is used and their services are not recognised; they complain that it keeps them from their work and they are not paid, nor is it reason for promotion.

- $\quad$ Health care interpreters require more than just linguistic knowledge; they need to have knowledge of medical terms but, more importantly, they need to have empathy and sensitivity for the doctor-patient relationship, an awareness of the role and responsibility of the interpreter in this relationship, and an ability to deal with patients' socio-cultural perspectives of health problems.

- The linguistic problems in the current situation include the fact that the informal isiXhosa interpreter may not always have adequate knowledge of English or Afrikaans, whereas others complain that they do not always understand the 'deep rural isiXhosa' of some patients.

The recommendations contained in the 
Emzantsi report include, among others, that health care interpreters require both professional language practitioner training and specific health interpreting. In the Western Cape, health care interpreters are likely to be newly recruited language practitioners, possibly with limited experience in interpreting. Along these lines one can argue for the need of accessible language use.

\section{An interpreter's perspective}

The most striking and challenging phenomenon in interpreting is its fundamental difficulty for the interpreter. Performance problems do not only occur in fast, information-dense or technical speeches, but also in clear, slow speech segments in which no particular obstacle can be detected (Gile 1995:159). These performance problems are exacerbated in the case of densely informative speeches or highly technical speeches, and are compounded by the possibility of insufficient understanding of the source language.

The effort models of Gile (1995) were developed to describe the interplay between different sets of cognitive operations involved in simultaneous interpreting (SI) and consecutive interpreting (CI). These sets of operations were grouped into 'efforts', which compete for a limited amount of processing capacity. The Listening and Analysing Effort (L) includes all reception and comprehension operations; the Memory Effort (M) designates the storing of information in the interpreter's short-term memory for the interval between the moment the speech is heard and the completion of its formulation; and the Production Effort $(\mathrm{P})$ represents all operations extending from the mental representation of the message to its actual formulation in the target language. These three efforts make demands on the interpreter's processing capacity at any time, together with a Coordination Effort (C), which represents the additional cognitive load required for managing the three efforts simultaneously. When the sum of the available capacity exceeds total requirement, the necessary cognitive balance between the efforts is disrupted, which results in failure sequences, with different errors and omissions. This is even more complex in the case of consecutive interpreting, in the sense that two phases can be distinguished. The first phase constitutes listening and analysis, note-taking, short-term memory and coordination, while the second constitutes remembering, note-reading and production. It becomes essential for the interpreter to balance these requirements, as he or she has only limited mental energy available for coordinating all these mental efforts.

The question that arises is: how can one assist the interpreter as an intercultural broker in the performance of his or her duties? One way of assisting in the case of liaison interpreting is via language use. It becomes essential that the service provider should use accessible, plain language and limit the use of a rich corpus or learned language. English has a very long history and a rich corpus that has developed over centuries. This corpus can rightfully be employed with great efficiency in different linguistic situations. But, once again, one should caution against the variables that could work against efficient communication if the corpus is used.

To make this corpus more digestible in a liaison interpreting context and to ensure that interpreting becomes more than a symbolic gesture, one will argue in favour of plain language - with all its limitations. What is plain language? Derrick Fine (2001: 19-21) describes plain language as clear, understandable, accessible and user friendly. It is therefore understandable and informative language, with a clear and well-organised structure, a clear and user-friendly layout and design for written materials, using visual back-up when speaking, and an appropriate and user-friendly tone and body language when speaking.

For readers and listeners at different levels, plain language means writing and speaking at a level that most people can understand. A plain language approach to communication for the sake of interpreting in a multicultural context requires that the service provider (professional) think of plain language as part of effective communication. It should be borne in mind that there are degrees or levels of plainness. What one usually achieves is a relative plainness, i.e. 'plainer' language that is an improvement on the original source language rather than perfectly plain language.

For the interpreter, there are basically two approaches: i) to put into plain language before interpreting, and ii) to put into plain language during interpreting. The latter approach requires more mental effort because it also entails rephrasing and adoption capabilities in order to ensure effective intercultural communication.

The danger of not using plain language in the case of interpreting is further complicated by the gravitational model of linguistic availability. This model of language proficiency is applicable to the lexicon, syntactic and other linguistic rules. It represents the status of the individual's oral or written command of a language at a particular time and in the particular circumstances by describing the relative availability of lexical units and linguistic rules.

The model consists of a variable and an invariable part. The latter refers to language elements of which the availability is assumed to be constant or to vary very slowly. This applies to the most basic rules of grammar and to a small number of the most frequently used words in the language. The variable part is much larger, as it includes dozens of rules and many thousands of words, idioms and metaphorical language. (Gile 1995: 216)

I want to argue that plain language is closer to the nucleus and that its lexical items and linguistic rules and structure belong to the invariable part. This means that plain language is more readily available for the interpreter to retrieve from his or her 'black box'. The dynamics of the gravitational model make provision for the interpreter to improve the availability of the linguistic material in his or her active zone. The underlying principle is that the more frequently words and rules are used, the stronger the centripetal effect will be, which means that words used very frequently become more available than words or rules used less frequently. The interpreter can better his or her production by ensuring that (difficult) language used frequently within a particular zone is readily available to him or her. Unfortunately, the problem is not solved if our aim is effective communication across cultural boundaries and the power gap barrier, simply because the client is not in a position to grasp the linguistic item immediately, even if he or she has come across it in the past. The opposite of the aforementioned principle applies in this 
regard, namely that the stimulated words and rules tend to drift outward (away from the centre of the system) and with that also the meaning thereof.

\section{Conclusion}

It seems clear that the interpreter has a pivotal role to play in a multilingual and multicultural situation. Unfortunately, it too often happens that the interpreter does not have a native speaker's competence in English - although the degree of competency obviously varies. It may be difficult for the interpreter to comprehend the question or to interpret it appropriately, whether in a (para) legal context (i.e. legal interpreting) or health context (i.e. liaison interpreting). Vital decisions that could result in fatal consequences will be made on the future or treatment of the client or patient based on the message being conveyed via the interpreter. Even if the interpreter does understand, the power and education gap still remains. Furthering the problem are dialectical differences and code mixing.

As a professional, one's language use is often a major barrier to effective communication. The availability of an interpreter in a multicultural setup to enhance effective communication is a step in the right direction. But the interpreter is a human being, with strengths and limitations. The act of interpreting is in itself a task that requires extreme mental energy to be available in the correct amount. It should also be borne in mind that the interpreter does not have the luxury of time at his or her disposal.

\section{Bibliography}

CENSUS IN BRIEF 2001: Statistics South Africa 2nd edition. South Africa, Pretoria.

COLE, P; LAWRENCE, L; NYUBUSE, N \& GODDEN, J. 2003: Costing the Western Cape language policy. Emzantsi Associates.

CRAWFORD, A 1994: Black patients/White doctors: Stories lost in translation. National Language Project occasional paper. Cape Town: National Language Project.

ERGUETA, E 1992: Hospital interpreting In: Jerome Quarterly. Washington: Georgetown University.

FINE, D 2001: "Plain language communication: Approaches and challenges." In: Viljoen, F and Nienaber, A (eds.), Pretoria: Protea Book House.

GILE, D 1995: Basic concepts and models for interpreter and translator training. Volume 8. Amsterdam/Philadelphia: John Benjamins Publishing Company.

FOURIE, J 2003: The quality of translation regarding medical research. Questionnaires. Unpublished MA Thesis, Stellenbosch: University of Stellenbosch.

LESCH, HM 1999: Gemeenskaps-vertaling in Suid-Afrika: die konteks van die ontvanger as normeringsbeginsel. Unpublished DLitt dissertation, Bellville: University of the Western Cape.

MULLER, L 1996: Poor clinical interpreting for 'good' psychiatric practice (?) $\underline{\text { In: }}$ Communication for the Health Professions in a multi-lingual society. Proceedings of a one day conference at the University of Natal, 11 July 1996, pp. 52-67.

NTSHONA, MS 1999: Towards a development of an interpreting model for the health sector in South Africa in Erasmus (ed) Liaison interpreting in the community. Goodwood: Van Schaik.

SCHLEMMER, AC 2005: Exploring the effects of a language barrier between the patients and staff at Hottentots Holland Hospital. Research assignment in part fulfilment of the M Fam Med Degree, University of Stellenbosch.

STATISTICS SOUTH AFRICA 1999: Provincial Profile Western Cape

SWARTZ, L 1992: Healing language gaps. Language Projects Review 7(3), pp. 11-13.

VILJOEN, F \& NIENABER, A (eds.) 2001: Plain language in South Africa: Report on an empirical research project. In: Viljoen, F and Nienaber, A. Plain legal language for a

\section{Addendum A - Distribution according to population group and level of education for people aged 20 and more}

\begin{tabular}{|l|l|l|l|l|l|}
\hline Education & Black/African & Coloured & Asian/Indian & White & Average \\
\hline No schooling & $22.3 \%$ & $8.3 \%$ & $5.3 \%$ & $1.4 \%$ & $17.9 \%$ \\
\hline Some primary & $18.5 \%$ & $18.4 \%$ & $7.7 \%$ & $1.2 \%$ & $16.0 \%$ \\
\hline Completed primary & $6.9 \%$ & $9.8 \%$ & $4.2 \%$ & $0.8 \%$ & $6.4 \%$ \\
\hline Some secondary & $30.4 \%$ & $40.1 \%$ & $33 \%$ & $25.9 \%$ & $30.8 \%$ \\
\hline Grade 12/ Std 10 & $16.8 \%$ & $18.5 \%$ & $34.9 \%$ & $40.9 \%$ & $20.4 \%$ \\
\hline Higher & $5.2 \%$ & $4.9 \%$ & $14.9 \%$ & $29.8 \%$ & $8.4 \%$ \\
\hline
\end{tabular}

(Source: Statistics South Africa, October Household Survey 1999) 
new democracy. Pretoria: Protea Book House.

YEKISO, NJ 2004: The African languages and the law: challenges and responses. University of Stellenbosch on 7 May 2004.

Addendum B - Population aged 20 years and older by highest level of education and population group and sex, Western Cape, 1999

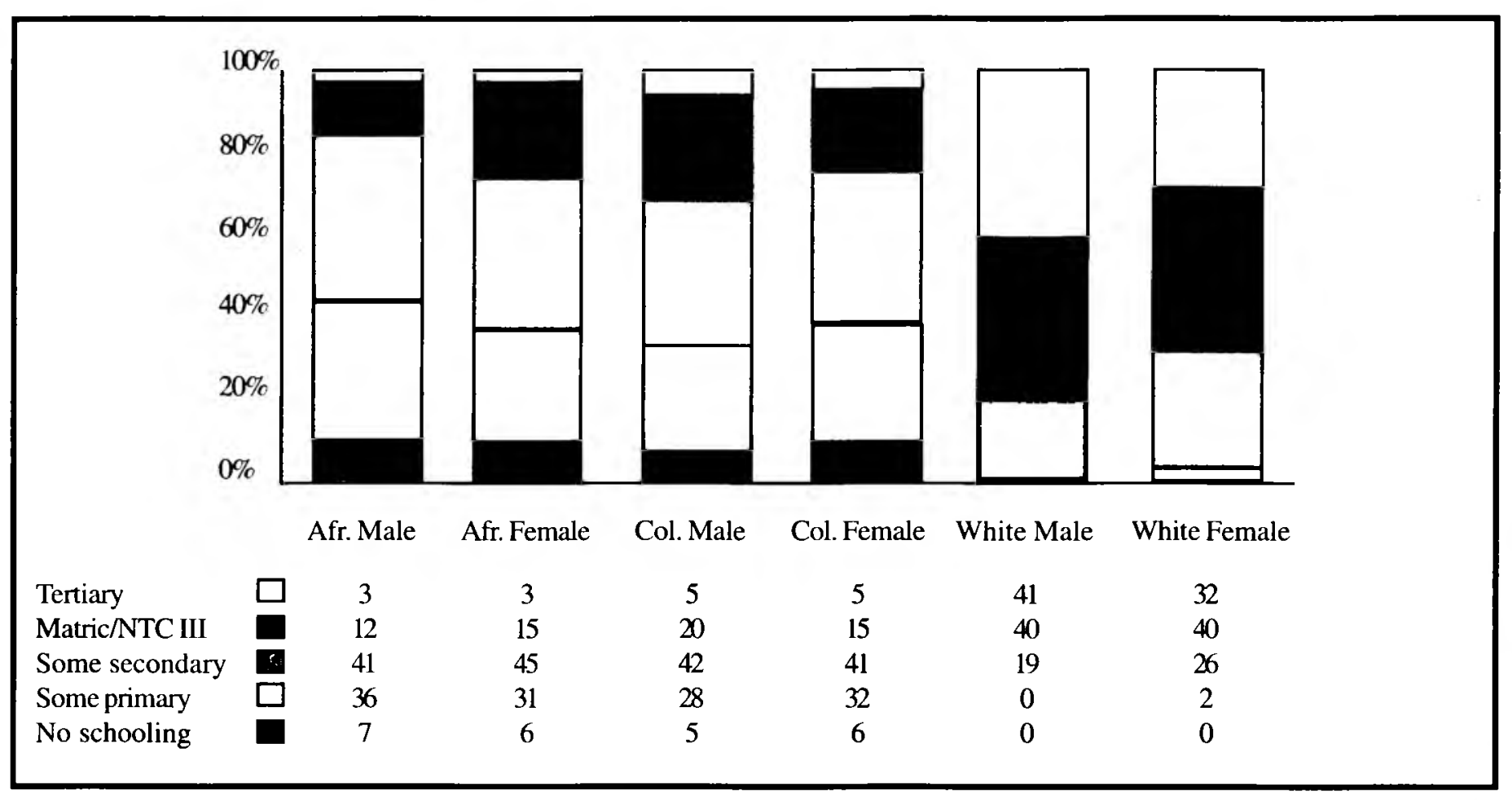

Source : Statistics South Africa, October Household Survey, 1999 